\begin{tabular}{|c|c|c|c|c|c|c|c|c|c|c|c|}
\hline Planet & \multicolumn{2}{|c|}{1905} & M. Z. Wien & Gr. & \multicolumn{2}{|r|}{$\alpha$ app. } & $\log p \cdot \Delta$ & \multicolumn{3}{|c|}{$\delta$ app. } & $\log p . \Delta$ \\
\hline $1905 \mathrm{QT}$ & Aug. & $\mathbf{x}$ & I $3^{h} 46^{m} 41^{s}$ & $3^{\mathrm{m}} \cdot \mathrm{o}$ & $23^{h}$ & $7^{\mathrm{m}} \cdot 34^{\mathrm{s}} \circ 7$ & $8.845 n$ & $-4^{\circ}$ & $35^{\circ}$ & $4 " .2$ & 0.843 \\
\hline I $905 \mathrm{QU}$ & Juli & 30 & $1425 \quad 53$ & $\mathrm{r} 2.5$ & 23 & 847.18 & $8.27 \circ_{n}$ & -3 & 43 & 26.6 & 0.838 \\
\hline D & Aug. & $\mathbf{I}$ & 358 & - & 23 & 814.39 & $9 \cdot 364 \mathrm{n}$ & -3 & $4 \mathrm{I}$ & 59.9 & 0.835 \\
\hline 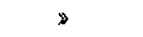 & » & 3 & $2 \quad 14$ & - & 23 & 732.67 & 9.1 I $5 n$ & -3 & 41 & $4 \cdot 3$ & 0.837 \\
\hline $1905 \mathrm{QV}$ & » & 3 & I $240 \quad 3$ I & 13.0 & 22 & I I 2 I. 63 & $8.873 n$ & -9 & 9 & 31.7 & 0.867 \\
\hline
\end{tabular}

1905 QT und QU sind neue, von mir entdeckte Planeten, Zur Sicherung, daß diese beiden nicht mit dem nahestehenden ( 167$)$ Urda identisch sind, ist der letztere ebenfalls beobachtet worden.

Wien, k. k. Sternwarte, 1905 Aug. 4.

F. Palisa.

\title{
Photographische Aufnahmen von kleinen Planeten 1905 Juli 30.
}

Objekt

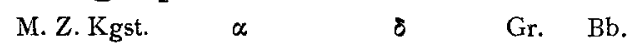

(263) Dresda

(241) Germania $13^{\mathrm{h}} 24^{\mathrm{m}} \mathrm{r}_{2}$ $22^{\mathrm{h}} 3^{\mathrm{m}} \cdot 9$ $-9^{\circ} 18^{\circ}$

I $3 \mathrm{~W}$

Objekt

M. Z. Kgst. $\begin{array}{ccccc}\alpha & \delta & \text { Gr. } & B b \\ 22^{\mathrm{h}} & \text { 1 } 0^{\mathrm{m}} 4 & -8^{\circ} 30^{\prime} & 12.4 & \text { W } \\ 22 & \text { 1 } 3.8 & -8 & 56 & \text { 1 } 2.8\end{array}$

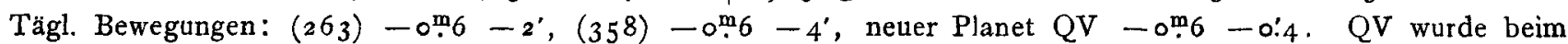
Suchen nach (260) Huberta gefunden, ist aber nicht mit diesem identisch.

Astrophys. Institut Königstuhl-Heidelberg, I905 Aug. 1.

M. Wolf.

Beobachtungen des Planeten (216) Kleopatra [1905 QR].

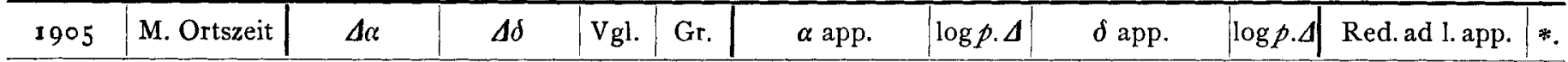

Auf der Sternwarte des Collegio Romano in Rom von Dr. E. Bianchi.

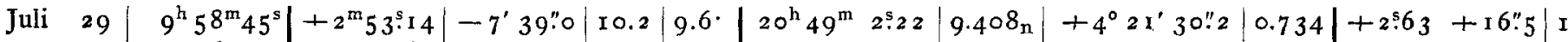

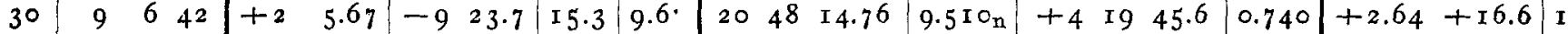

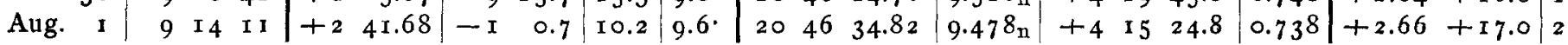

Auf der Sternwarte in Düsseldorf von Dr. $W$. Luther.

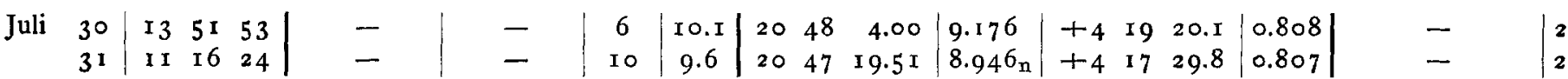

Die tägl. Bewegung des Planeten ist in Dekl. südlich, nicht nördlich, wie im Telegramm A. N. 4037 angegeben.

$$
\begin{aligned}
& \text { Mittlere Örter der Vergleichsterne. } \\
& \alpha 1905.0 \quad 81905.0 \quad \text { Autorität } \\
& \text { I } 20^{\mathrm{h}} 46^{\mathrm{m}} 6^{\mathrm{s}} .45+4^{\circ}{ }_{2} 8^{\prime} 5^{\mathrm{l}} .7 \quad 1 / 2 \text { (Toul. IV, } 3230+\text { Vf. Bonn 4) } \\
& 2 \quad 204350.48+4 \text { I6 } 8.5 \quad \text { AG. Albany } 7277
\end{aligned}
$$

\section{Une nouvelle variable du type Algol 79.1905 Cephei.}

Le 7 Février 1905 , Mme. L. Ceraski a trouvé sur des plaques dues à $\mathrm{M}$. S. Blajko une nouvelle variable qui sur 20 clichés était toujours de $9.5 \mathrm{gr}$. environ et sur un seulement, obtenu en 1904 octobre $1,7^{\mathrm{h}} 24^{\mathrm{m}}-9^{\mathrm{h}} 24^{\mathrm{m}}$ t. $\mathrm{m}$. de Moscou, elle était de beaucoup plus faible $\left(12^{\circ} \mathrm{gr}\right.$. à peu près).

Une seule plaque accusant la variabilité il a été décidé de ne pas la publier immédiatement. Depuis ce temps-là, M. S. Blajko a plus d'une fois observé cette étoile, mais la trouvait toujours de même éclat (à peu près de $9^{1 / 2}$ gr.); enfin, ce 14 juillet, il la vit de $12^{\theta} \mathrm{gr}$. environ; I 905 le r 9 juillet / I aout. de $10^{\mathrm{h}} 5^{\mathrm{m}}$ à $13^{\mathrm{h}} 14^{\mathrm{m}}$ elle demeura à peu près invariable. La période en est inconnue.

Voici la position approchée de notre étoile:

$$
\begin{array}{rrr}
\mathrm{r} 855 \alpha= & 4^{\mathrm{h}} 40^{\mathrm{m}} 5^{\mathrm{s}} \\
1900 & 44834
\end{array} \quad \delta=\begin{aligned}
& +80^{\circ} \mathrm{r}^{\mathrm{s}}: 0 \\
&
\end{aligned}
$$

Il est aisé de reconnaitre la variable d'après trois faibles étoiles qui la suivent et qui ont ces coordonnées relatives:

$$
\begin{array}{lrrr}
\Delta a \cos \delta & +2.1 & +5.8 & +6: 0 \\
\Delta \delta & -0.2 & 0.0 & -2.9
\end{array}
$$

Prof. W. Ceraski.

Inhalt zu Nr. 4039. A. Abetti. Asteroidi osservati ad Arcetri nel Ig04. 97. - H. Rosenberg. Helles Meteor von I904 März 21. Io5. $M$. und $G$. Wolf. Die Veränderlichen 78.1905 Virginis und Y Virginis. 107. - 7. Palisa. Beobachtungen von kleinen Planeten. Iog. - M. Wolf. Photographische Aufnahmen von kleinen Planeten I905 Juli 30. I I I. - Beobachtungen des Planeten (2I6) Kleopatra [1905 QR]. III. - W. Ceraski. Une nouvelle variable da type Algol 79.Ig05 Cephei. III. 